Bull. Korean Math. Soc. 48 (2011), No. 1, pp. 51-65

DOI 10.4134/BKMS.2011.48.1.051

\title{
CLASS-MAPPING PROPERTIES \\ OF THE HOHLOV OPERATOR
}

\author{
Akshaya K. Mishra and Trailokya Panigrahi
}

\begin{abstract}
In the present paper sufficient conditions, in terms of hypergeometric inequalities, are found so that the Hohlov operator preserves a certain subclass of close-to-convex functions (denoted by $\mathcal{R}^{\tau}(A, B)$ ) and transforms the classes consisting of $k$-uniformly convex functions, $k$-starlike functions and univalent starlike functions into $\mathcal{R}^{\tau}(A, B)$
\end{abstract}

\section{Introduction and definitions}

Let $\mathcal{A}_{0}$ be the class of analytic functions in the open unit disc

$$
\mathcal{U}:=\{z \in \mathbb{C}:|z|<1\}
$$

and having the normalized power series expansion

$$
f(z)=z+\sum_{n=2}^{\infty} a_{n} z^{n}(z \in \mathcal{U}) .
$$

The class $\mathcal{S}$ consists of univalent functions in $\mathcal{A}_{0}$. The function $f \in \mathcal{A}_{0}$ is said to be in $k-\mathcal{U C V}$, the class of $k$-uniformly convex functions $(0 \leq k<\infty)$, if $f \in \mathcal{S}$ along with the property that for every circular arc $\gamma$ contained in $\mathcal{U}$, with center $\zeta$ where $|\zeta| \leq k$, the image curve $f(\gamma)$ is a convex arc (cf. [10]). It is well known that (see [10]) $f \in k-\mathcal{U C V}$ if and only if the image of the function $p$, where

$$
p(z)=1+\frac{z f^{\prime \prime}(z)}{f^{\prime}(z)}(z \in \mathcal{U}),
$$

is a subset of the conic region

$$
\Omega_{k}:=\left\{w=u+i v: u^{2}>k^{2}(u-1)^{2}+k^{2} v^{2}, 0 \leq k<\infty\right\} .
$$

Received May 10, 2009.

2010 Mathematics Subject Classification. 30C45, 33E05.

Key words and phrases. univalent, $k$-uniformly convex, parabolic starlike, hypergeometric series, Hadamard product, Hohlov operator. 
The class $k-\mathcal{S T}$, consisting of $k$-starlike functions, is defined via $k-\mathcal{U C V}$ by the usual Alexander's relation, i.e.,

$$
f \in k-\mathcal{S T} \Longleftrightarrow g \in k-\mathcal{U C V} \text {, where } g(z)=\int_{0}^{z}\left(\frac{f(t)}{t}\right) d t \text { (see e.g. [11]). }
$$

In particular, if $k=0$ and $k=1$, we get

$$
0-\mathcal{U C V} \equiv \mathcal{C} \mathcal{V}, 0-\mathcal{S} \mathcal{T} \equiv \mathcal{S T}, 1-\mathcal{U C} \mathcal{V} \equiv \mathcal{U C V} \text { and } 1-\mathcal{S} \mathcal{T} \equiv \mathcal{S P}
$$

where $\mathcal{C V}, \mathcal{S T}, \mathcal{U C V}$, and $\mathcal{S P}$ are respectively the familiar classes of univalent convex functions, univalent starlike functions [4], uniformly convex functions ([7], also see [12], [15]) and parabolic starlike functions [15]. For a unified and systematic study of these classes with the aid of fractional calculus, see e.g. $[17,18,19,20,21]$.

The function $f \in \mathcal{A}_{0}$ is said to be in the class $\mathcal{R}^{\tau}(A, B)$ (see [3]) if

$$
\left|\frac{f^{\prime}(z)-1}{(A-B) \tau-B\left(f^{\prime}(z)-1\right)}\right|<1(z \in \mathcal{U}, \tau \in \mathbb{C} \backslash\{0\},-1 \leq B<A \leq 1) .
$$

For particular values of $A, B$ and $\tau$ the class $\mathcal{R}^{\tau}(A, B)$ includes certain interesting subclasses of $\mathcal{S}$. For example, by taking

$$
\tau=e^{-i \eta} \cos \eta\left(-\frac{\pi}{2}<\eta<\frac{\pi}{2}\right), A=1-2 \beta(0 \leq \beta<1) \text { and } B=-1
$$

we get the class $\mathcal{R}_{\eta}(\beta)$, studied by Ponnusamy and Ronning [14], where

$$
\mathcal{R}_{\eta}(\beta)=\left\{f \in \mathcal{A}_{0}: \Re\left(e^{i \eta}\left(f^{\prime}(z)-\beta\right)\right)>0, z \in \mathcal{U},-\frac{\pi}{2}<\eta<\frac{\pi}{2}, 0 \leq \beta<1\right\} .
$$

Similarly, if we set $\tau=1, A=\beta, B=-\beta(0<\beta \leq 1)$ we obtain the class of functions $f \in \mathcal{A}_{0}$ satisfying the inequality

$$
\left|\frac{f^{\prime}(z)-1}{f^{\prime}(z)+1}\right|<\beta(z \in \mathcal{U}, 0<\beta \leq 1)
$$

studied earlier by Padmanabhan [13], Caplinger and Causey [2] and others. Note that the functions in the class $\mathcal{R}^{\tau}(A, B)$ are univalent and close-to-convex.

The generalized hypergeometric function ${ }_{p} F_{q}\left(p, q \in \mathbb{N}_{0}:=\{0,1,2, \ldots\}\right)$ with $p$ numerator parameters $\alpha_{j} \in \mathbb{C}(j=1, \ldots, p)$ and $q$ denominator parameters $\beta_{k} \in \mathbb{C} \backslash \mathbb{Z}_{0}^{-}\left(\mathbb{Z}_{0}^{-}:=\{0,-1,-2, \ldots\}, k=1, \ldots, q\right)$; is defined by (cf. [16])

$$
{ }_{p} F_{q}(z)={ }_{p} F_{q}\left(\alpha_{1}, \ldots, \alpha_{p} ; \beta_{1}, \ldots, \beta_{q} ; z\right):=\sum_{n=0}^{\infty} \frac{\left(\alpha_{1}\right)_{n} \cdots\left(\alpha_{p}\right)_{n}}{\left(\beta_{1}\right)_{n} \cdots\left(\beta_{q}\right)_{n}} \frac{z^{n}}{n !},
$$

where $(\lambda)_{n}$ is the Pochhammer symbol (or shifted factorial), defined in terms of the gamma function by

$$
(\lambda)_{n}:=\frac{\Gamma(\lambda+n)}{\Gamma(\lambda)}=\left\{\begin{array}{l}
1(n=0) \\
\lambda \cdots(\lambda+n-1)(n \in \mathbb{N}:=\{1,2, \ldots\}) .
\end{array}\right.
$$


Note that ${ }_{p} F_{q}(z)$ is an entire function if $p<q+1$. However, if $p=q+1$, then ${ }_{p} F_{q}(z)$ is analytic in $\mathcal{U}$. Also, if

$$
p=q+1 \text { and } \Re\left(\sum_{j=1}^{q} \beta_{j}-\sum_{j=1}^{p} \alpha_{j}\right)>0,
$$

then ${ }_{p} F_{q}(z)$ converges on $\partial \mathcal{U}$. In particular, the function

$$
{ }_{2} F_{1}(a, b ; c ; z):=\sum_{n=0}^{\infty} \frac{(a)_{n}(b)_{n}}{(c)_{n}(1)_{n}} z^{n}
$$

is the familiar Gaussian hypergeometric function. Furthermore, the evaluation ${ }_{2} F_{1}(a, b ; c ; 1)$ is related to the gamma function by

$$
{ }_{2} F_{1}(a, b ; c ; 1)=\frac{\Gamma(c) \Gamma(c-a-b)}{\Gamma(c-a) \Gamma(c-b)}\left(\Re(c-a-b)>0, c \notin \mathbb{Z}_{0}^{-}\right) .
$$

We now recall the Hohlov operator $\mathcal{I}_{c}^{a, b}: \mathcal{A}_{0} \rightarrow \mathcal{A}_{0}$, defined in terms of the Hadamard product (or convolution) by (cf. [8])

$$
\left(\mathcal{I}_{c}^{a, b}(f)\right)(z)=z_{2} F_{1}(a, b ; c ; z) * f(z)\left(f \in \mathcal{A}_{0}, z \in \mathcal{U}\right) .
$$

Thus from (1.1) and (1.4) we have

$$
\left(\mathcal{I}_{c}^{a, b}(f)\right)(z)=z+\sum_{n=2}^{\infty} \frac{(a)_{n-1}(b)_{n-1}}{(c)_{n-1}(1)_{n-1}} a_{n} z^{n}(z \in \mathcal{U}) .
$$

It is well known that the class $\mathcal{S}$ and many of its important subclasses are not closed under the ring operations of usual addition and multiplication of functions. Therefore, the study of class-preserving and class-transforming operators is an interesting problem in geometric function theory. The Hohlov operator unifies several such previously well studied operators, namely the Alexander, Libera, Bernardi and Carlson-Shaffer operators (denoted here by $\mathcal{A}, \mathcal{L}, \mathcal{B}$ and $\mathcal{L}(a, c)$ respectively). Thus

$$
\mathcal{A}(f)=\mathcal{I}_{2}^{1,1}(f), \mathcal{L}(f)=\mathcal{I}_{3}^{1,2}(f), \mathcal{B}(f)=\mathcal{I}_{\gamma+2}^{1, \gamma+1}(f), \mathcal{L}(a, c)(f)=\mathcal{I}_{c}^{a, 1}(f) .
$$

Kanas and Srivastava [9] and Ponnusamy and Ronning [14] (also see Gangadharan et al. [5]) obtained coefficient inequalities so that the operator $I_{c}^{a, b}$ preserves the class $k-\mathcal{U C V}$ and transforms the classes

$$
\mathcal{R}_{\eta}(\beta) \text { into } k-\mathcal{U C V} ; R_{\eta}(\beta) \text { into } k-\mathcal{S T} ;
$$

$\mathcal{S T}$ into $k-\mathcal{U C V} ; \mathcal{S T}$ into $k-\mathcal{S T}$ and $k-\mathcal{U C V}$ into $k-\mathcal{S T}$.

The main object of the present paper is to consider the more general class $\mathcal{R}^{\tau}(A, B)$ (instead of $\mathcal{R}_{\eta}(\beta)$ ) and find sufficient conditions in terms of hypergeometric inequalities for the reverse of some of the transformations considered in [9] and [14]. More specifically sufficient conditions are obtained here to ensure that the Hohlov operator $\mathcal{I}_{c}^{a, b}$ maps the classes

$$
k-\mathcal{U C V} \text { into } \mathcal{R}^{\tau}(A, B), k-\mathcal{S} \mathcal{T} \text { into } \mathcal{R}^{\tau}(A, B) \text { and } \mathcal{S} \mathcal{T} \text { into } \mathcal{R}^{\tau}(A, B)
$$


Furthermore, the invariance of the class $\mathcal{R}^{\tau}(A, B)$ under the operator $\mathcal{I}_{c}^{a, b}$ is discussed. Lastly, a sufficient condition is obtained so that the function $z_{2} F_{1}(a, b ; c ; z)$ belongs to $\mathcal{R}^{\tau}(A, B)$. Sufficient conditions for the particular cases of $\mathcal{I}_{c}^{a, b}$ are also emphasized in the form of corollaries to the main theorems.

\section{Some preliminary lemmas}

We need each of the following results in our investigation.

Lemma 1 (see [10], [11]). Let

$$
P_{k}(z)=1+p_{1}(k) z+p_{2}(k) z^{2}+\cdots\left(z \in \mathcal{U}, p_{1}(k)>0\right)
$$

be the Riemann map of $\mathcal{U}$ onto $\Omega_{k}$ where the region $\Omega_{k}$ is defined as in (1.2) and let the function $f$ be given by (1.1). If $f \in k-\mathcal{U C V}$, then

$$
\left|a_{n}\right| \leq \frac{\left(p_{1}(k)\right)_{n-1}}{n !}(n \in \mathbb{N} \backslash\{1\}) .
$$

Further if $f \in k-\mathcal{S T}$, then

$$
\left|a_{n}\right| \leq \frac{\left(p_{1}(k)\right)_{n-1}}{(n-1) !}(n \in \mathbb{N} \backslash\{1\}) .
$$

The estimates (2.2) and (2.3) are sharp.

Lemma 2 (see [3]). Let the function $f$, given by $(1.1)$, be a member of $\mathcal{R}^{\tau}(A, B)$. Then

$$
\left|a_{n}\right| \leq(A-B) \frac{|\tau|}{n}(n \in \mathbb{N} \backslash\{1\}) .
$$

The estimate in (2.4) is sharp for the function

$$
f(z)=\int_{0}^{1}\left(1+\frac{(A-B) \tau t^{n-1}}{1+B t^{n-1}}\right) d t(z \in \mathcal{U}, n \in \mathbb{N} \backslash\{1\}) .
$$

Lemma 3 (see [3]). Let the function $f \in \mathcal{A}_{0}$ be of the form (1.1). If

$$
\sum_{n=2}^{\infty}(1+|B|) n\left|a_{n}\right| \leq(A-B)|\tau|(-1 \leq B<A \leq 1, \tau \in \mathbb{C} \backslash\{0\}),
$$

then $f \in \mathcal{R}^{\tau}(A, B)$. The result is sharp for the function

$$
f(z)=z+\frac{(A-B) \tau}{(1+|B|) n} z^{n}(z \in \mathcal{U}, n \in \mathbb{N} \backslash\{1\}) .
$$

Lemma 4 (see [1]). Let the function $f$ of the form (1.1) be a member of $\mathcal{S}$ (or $\mathcal{S} \mathcal{T}$ ). Then the sharp estimate

$$
\left|a_{n}\right| \leq n(n \in \mathbb{N} \backslash\{1\})
$$

holds true. 
Lemma 5 (see [6]). Let the function $f \in \mathcal{A}_{0}$ be of the form (1.1). If

$$
\sum_{n=2}^{\infty} n\left|a_{n}\right| \leq 1
$$

then $f \in \mathcal{S T}$.

\section{Mapping properties of the Hohlov operator}

Throughout in the present section we shall take

$$
-1 \leq B<A \leq 1, \frac{-\pi}{2}<\eta<\frac{\pi}{2} .
$$

Theorem 1. Let $a, b \in \mathbb{C} \backslash\{0\}$ and $c \in \mathbb{C}$ satisfy

$$
\Re c>\max \{0,2 \Re a+2,2 \Re b+2\} .
$$

If the hypergeometric inequality

$$
\begin{aligned}
& \quad \frac{\Gamma(\Re c)\{\Gamma(\Re c-2 \Re a-2) \Gamma(\Re c-2 \Re b-2)\}^{\frac{1}{2}}\left[\left|(a)_{2}\right|\left|(b)_{2}\right|\right.}{|\Gamma(\Re c-a)||\Gamma(\Re c-b)|} \\
& \quad+3|a b|\{(\Re c-2 \Re a-2)(\Re c-2 \Re b-2)\}^{\frac{1}{2}} \\
& \left.\quad+\left\{(\Re c-2 \Re a-2)_{2}(\Re c-2 \Re b-2)_{2}\right\}^{\frac{1}{2}}\right] \\
& \leq \frac{(A-B)|\tau|}{(1+|B|)}+1
\end{aligned}
$$

is satisfied, then $\mathcal{I}_{c}^{a, b}$ maps the class $\mathcal{S}$ (or $\left.\mathcal{S} \mathcal{T}\right)$ into $\mathcal{R}^{\tau}(A, B)$.

Proof. Let the function $f$ given by $(1.1)$ be a member of $\mathcal{S}$ or $\mathcal{S T}$. By $(1.7)$

$$
\mathcal{I}_{c}^{a, b}(f)=z+\sum_{n=2}^{\infty} \frac{(a)_{n-1}(b)_{n-1}}{(c)_{n-1}(1)_{n-1}} a_{n} z^{n}(z \in \mathcal{U}) .
$$

In view of Lemma 3 , it is thus sufficient to show that

$$
\sum_{n=2}^{\infty}(1+|B|) n\left|\frac{(a)_{n-1}(b)_{n-1}}{(c)_{n-1}(1)_{n-1}} a_{n}\right| \leq(A-B)|\tau| .
$$

By making use of Lemma 4 and the elementary inequality

$$
\left|(c)_{p}\right|>(\Re c)_{p}(p \in \mathbb{N})
$$

it is again sufficient to prove that

$$
S_{1}=\sum_{n=2}^{\infty} n^{2} \frac{\left|(a)_{n-1}(b)_{n-1}\right|}{(\Re c)_{n-1}(1)_{n-1}} \leq \frac{(A-B)|\tau|}{(1+|B|)} .
$$

The term $S_{1}$ above is equivalently written as

$$
S_{1}=\sum_{n=1}^{\infty}(n+1)^{2} \frac{\left|(a)_{n}(b)_{n}\right|}{(\Re c)_{n}(1)_{n}}=\sum_{n=1}^{\infty}\{n(n-1)+3 n+1\} \frac{\left|(a)_{n}(b)_{n}\right|}{(\Re c)_{n}(1)_{n}}
$$




$$
\begin{aligned}
& =\sum_{n=2}^{\infty} \frac{\left|(a)_{n}(b)_{n}\right|}{(\Re c)_{n}(1)_{n-2}}+3 \sum_{n=1}^{\infty} \frac{\left|(a)_{n}(b)_{n}\right|}{(\Re c)_{n}(1)_{n-1}}+\sum_{n=1}^{\infty} \frac{\left|(a)_{n}(b)_{n}\right|}{(\Re c)_{n}(1)_{n}} \\
& =\sum_{n=0}^{\infty} \frac{\left|(a)_{n+2}\right|\left|(b)_{n+2}\right|}{(\Re c)_{n+2}(1)_{n}}+3 \sum_{n=0}^{\infty} \frac{\left|(a)_{n+1}\right|\left|(b)_{n+1}\right|}{(\Re c)_{n+1}(1)_{n}}+\sum_{n=0}^{\infty} \frac{\left|(a)_{n}\right|\left|(b)_{n}\right|}{(\Re c)_{n}(1)_{n}}-1 .
\end{aligned}
$$

The repeated applications of the relation

$$
(d)_{m}=d(d+1)_{m-1}(d \in \mathbb{C}, m \in \mathbb{N})
$$

yield

$$
\begin{aligned}
S_{1}= & \frac{\left|(a)_{2}\right|\left|(b)_{2}\right|}{(\Re c)_{2}} \sum_{n=0}^{\infty} \frac{\left|(a+2)_{n}\right|\left|(b+2)_{n}\right|}{(\Re c+2)_{n}(1)_{n}} \\
& +\frac{3|a b|}{\Re c} \sum_{n=0}^{\infty} \frac{\left|(a+1)_{n}\right|\left|(b+1)_{n}\right|}{(\Re c+1)_{n}(1)_{n}}+\sum_{n=0}^{\infty} \frac{\left|(a)_{n}\right|\left|(b)_{n}\right|}{(\Re c)_{n}(1)_{n}}-1 .
\end{aligned}
$$

Applying Cauchy's inequality to individual sums in (3.5) we get

$$
\begin{aligned}
S_{1} \leq & \frac{\left|(a)_{2}\right|\left|(b)_{2}\right|}{(\Re c)_{2}}\left[\left\{\sum_{n=0}^{\infty} \frac{(a+2)_{n}(\bar{a}+2)_{n}}{(\Re c+2)_{n}(1)_{n}}\right\}^{\frac{1}{2}}\left\{\sum_{n=0}^{\infty} \frac{(b+2)_{n}(\bar{b}+2)_{n}}{(\Re c+2)_{n}(1)_{n}}\right\}^{\frac{1}{2}}\right] \\
& +\frac{3|a b|}{\Re c}\left[\left\{\sum_{n=0}^{\infty} \frac{(a+1)_{n}(\bar{a}+1)_{n}}{(\Re c+1)_{n}(1)_{n}}\right\}^{\frac{1}{2}}\left\{\sum_{n=0}^{\infty} \frac{(b+1)_{n}(\bar{b}+1)_{n}}{(\Re c+1)_{n}(1)_{n}}\right\}^{\frac{1}{2}}\right] \\
& +\left[\left\{\sum_{n=0}^{\infty} \frac{(a)_{n}(\bar{a})_{n}}{(\Re c)_{n}(1)_{n}}\right\}^{\frac{1}{2}}\left\{\sum_{n=0}^{\infty} \frac{(b)_{n}(\bar{b})_{n}}{(\Re c)_{n}(1)_{n}}\right\}^{\frac{1}{2}}\right]-1 \\
= & \frac{\left|(a)_{2}\right|\left|(b)_{2}\right|}{(\Re c)_{2}}\left[\left\{{ }_{2} F_{1}(a+2, \bar{a}+2 ; \Re c+2 ; 1)\right\}^{\frac{1}{2}}\left\{{ }_{2} F_{1}(b+2, \bar{b}+2 ; \Re c+2 ; 1)\right\}^{\frac{1}{2}}\right] \\
& +\frac{3|a b|}{\Re c}\left\{{ }_{2} F_{1}(a+1, \bar{a}+1 ; \Re c+1 ; 1)\right\}^{\frac{1}{2}}\left\{{ }_{2} F_{1}(b+1, \bar{b}+1 ; \Re c+1 ; 1)\right\}^{\frac{1}{2}} \\
& +\left\{{ }_{2} F_{1}(a, \bar{a} ; \Re c ; 1)\right\}^{\frac{1}{2}}\left\{{ }_{2} F_{1}(b, \bar{b} ; \Re c ; 1)\right\}^{\frac{1}{2}}-1 .
\end{aligned}
$$

Since the condition (3.1) holds we use the Gauss summation formula (1.5) and get

$$
\begin{aligned}
S_{1} \leq & \frac{\left|(a)_{2}\right|\left|(b)_{2}\right|}{(\Re c)_{2}}\left\{\frac{\Gamma(\Re c+2) \Gamma(\Re c-2 \Re a-2)}{\Gamma(\Re c-a) \Gamma(\Re c-\bar{a})}\right\}^{\frac{1}{2}}\left\{\frac{\Gamma(\Re c+2) \Gamma(\Re c-2 \Re b-2)}{\Gamma(\Re c-b) \Gamma(\Re c-\bar{b})}\right\}^{\frac{1}{2}} \\
& +\frac{3|a b|}{\Re c}\left\{\frac{\Gamma(\Re c+1) \Gamma(\Re c-2 \Re a-1)}{\Gamma(\Re c-a) \Gamma(\Re c-\bar{a})}\right\}^{\frac{1}{2}}\left\{\frac{\Gamma(\Re c+1) \Gamma(\Re c-2 \Re b-1)}{\Gamma(\Re c-b) \Gamma(\Re c-\bar{b})}\right\}^{\frac{1}{2}} \\
& +\left\{\frac{\Gamma(\Re c) \Gamma(\Re c-2 \Re a)}{\Gamma(\Re c-a) \Gamma(\Re c-\bar{a})}\right\}^{\frac{1}{2}}\left\{\frac{\Gamma(\Re c) \Gamma(\Re c-2 \Re b)}{\Gamma(\Re c-b) \Gamma(\Re c-\bar{b})}\right\}^{\frac{1}{2}}-1 .
\end{aligned}
$$


Moreover, the gamma function is symmetric about real axis, i.e., $\overline{\Gamma(z)}=\Gamma(\bar{z})$. Therefore,

$$
\begin{aligned}
S_{1} \leq & \frac{\Gamma(\Re c)\{\Gamma(\Re c-2 \Re a-2) \Gamma(\Re c-2 \Re b-2)\}^{\frac{1}{2}}}{|\Gamma(\Re c-a)||\Gamma(\Re c-b)|}\left[\left|(a)_{2}\right|\left|(b)_{2}\right|\right. \\
& +3|a b|\{(\Re c-2 \Re a-2)(\Re c-2 \Re b-2)\}^{\frac{1}{2}} \\
& \left.+\left\{(\Re c-2 \Re a-2)_{2}(\Re c-2 \Re b-2)_{2}\right\}^{\frac{1}{2}}\right]-1 .
\end{aligned}
$$

Thus in view of (3.4) if the hypergeometric inequality (3.2) is satisfied, then $\mathcal{I}_{c}^{a, b}(f) \in \mathcal{R}^{\tau}(A, B)$ as asserted. The proof of Theorem 1 is complete.

Corollary 1. Let $a \in \mathbb{C} \backslash\{0\}$ and $c \in \mathbb{C}$ satisfy

$$
\Re c>\max \{0,2 \Re a+2\} .
$$

If the hypergeometric inequality

$$
\begin{aligned}
& \frac{\Gamma(\Re c) \Gamma(\Re c-2 \Re a-2)}{|\Gamma(\Re c-a)|^{2}}\left[\left|(a)_{2}\right|^{2}+3|a|^{2}(\Re c-2 \Re a-2)+(\Re c-2 \Re a-2)_{2}\right] \\
\leq & \frac{(A-B)|\tau|}{1+|B|}+1
\end{aligned}
$$

is satisfied, then $\mathcal{I}_{c}^{a, \bar{a}}$ maps the class $\mathcal{S}$ or $\mathcal{S} \mathcal{T}$ into $\mathcal{R}^{\tau}(A, B)$.

Proof. Take $b=\bar{a}$ in Theorem 1 .

Corollary 2. Let $a \in \mathbb{C} \backslash\{0\}$ and $c \in \mathbb{C}$ satisfy

$$
\Re c>\max \{4,2 \Re a+2\} .
$$

If the hypergeometric inequality

$$
\begin{aligned}
& \frac{\Gamma(\Re c)\{\Gamma(\Re c-2 \Re a-2) \Gamma(\Re c-4)\}^{\frac{1}{2}}}{|\Gamma(\Re c-a)| \Gamma(\Re c-1)}\left[2\left|(a)_{2}\right|\right. \\
& \left.+3|a|\{(\Re c-2 \Re a-2)(\Re c-4)\}^{\frac{1}{2}}+\left\{(\Re c-2 \Re a-2)_{2}(\Re c-4)_{2}\right\}^{\frac{1}{2}}\right] \\
\leq & \frac{(A-B)|\tau|}{1+|B|}+1
\end{aligned}
$$

is satisfied, then $\mathcal{L}(a, c)$ maps the class of $\mathcal{S}($ or $\mathcal{S} T)$ into $\mathcal{R}^{\tau}(A, B)$.

Proof. Take $b=1$ in Theorem 1.

Theorem 2. Let $a, b \in \mathbb{C} \backslash\{0\}, p_{1}=p_{1}(k)$ be defined by $(2.1)$ and $c \in \mathbb{C}$ satisfy

$$
\Re c>\max \left\{0,2 \Re a+p_{1}, 2 \Re b+p_{1}\right\} .
$$

(i) If the hypergeometric inequality

$$
\frac{|a b| p_{1}}{\Re c}\left\{{ }_{3} F_{2}\left(a+1, \bar{a}+1, p_{1}+1 ; \Re c+1,2 ; 1\right)\right\}^{\frac{1}{2}}\left\{{ } _ { 3 } F _ { 2 } \left(b+1, \bar{b}+1, p_{1}+1 ;\right.\right.
$$




$$
\begin{aligned}
& \Re c+1,2 ; 1)\}^{\frac{1}{2}}+\left\{{ }_{3} F_{2}\left(a, \bar{a}, p_{1} ; \Re c, 1 ; 1\right)\right\}^{\frac{1}{2}}\left\{{ }_{3} F_{2}\left(b, \bar{b}, p_{1} ; \Re c, 1 ; 1\right)\right\}^{\frac{1}{2}} \\
\leq & \frac{(A-B)|\tau|}{1+|B|}+1
\end{aligned}
$$

is satisfied, then $\mathcal{I}_{c}^{a, b}$ maps the class $k-\mathcal{S} \mathcal{T}$ into $\mathcal{R}^{\tau}(A, B)$.

(ii) Furthermore, if

$$
\begin{aligned}
& \frac{|a b| p_{1}}{\Re c}\left\{{ }_{3} F_{2}\left(a+1, \bar{a}+1, p_{1}+1 ; \Re c+1,2 ; 1\right)\right\}^{\frac{1}{2}}\left\{{ } _ { 3 } F _ { 2 } \left(b+1, \bar{b}+1, p_{1}+1 ;\right.\right. \\
& \Re c+1,2 ; 1)\}^{\frac{1}{2}}+\left\{{ }_{3} F_{2}\left(a, \bar{a}, p_{1} ; \Re c, 1 ; 1\right)\right\}^{\frac{1}{2}}\left\{{ }_{3} F_{2}\left(b, \bar{b}, p_{1} ; \Re c, 1 ; 1\right)\right\}^{\frac{1}{2}} \leq 2
\end{aligned}
$$

is satisfied, then $\mathcal{I}_{c}^{a, b}$ maps the class $k-\mathcal{S T}$ into $\mathcal{S T}$.

Proof. (i) Let the function $f$ given by (1.1) be a member of $k-\mathcal{S T}$. As in the proof of Theorem1, it is sufficient to show that

$$
\sum_{n=2}^{\infty}(1+|B|) n\left|\frac{(a)_{n-1}(b)_{n-1}}{(c)_{n-1}(1)_{n-1}} a_{n}\right| \leq(A-B)|\tau| .
$$

Using the coefficient estimate (2.3) it is again sufficient to show that

$$
S_{2}=\sum_{n=2}^{\infty} n \frac{\left|(a)_{n-1}(b)_{n-1}\right|\left(p_{1}\right)_{n-1}}{(\Re c)_{n-1}(1)_{n-1}(1)_{n-1}} \leq \frac{(A-B)|\tau|}{(1+|B|)} .
$$

Now

$$
\begin{aligned}
S_{2} & =\sum_{n=1}^{\infty}(n+1) \frac{\left|(a)_{n}(b)_{n}\right|\left(p_{1}\right)_{n}}{(\Re c)_{n}(1)_{n}(1)_{n}}=\sum_{n=1}^{\infty} \frac{\left|(a)_{n}(b)_{n}\right|\left(p_{1}\right)_{n}}{(\Re c)_{n}(1)_{n-1}(1)_{n}}+\sum_{n=1}^{\infty} \frac{\left|(a)_{n}(b)_{n}\right|\left(p_{1}\right)_{n}}{(\Re c)_{n}(1)_{n}(1)_{n}} \\
& =\frac{|a b| p_{1}}{\Re c} \sum_{n=0}^{\infty} \frac{\left|(a+1)_{n}(b+1)_{n}\right|\left(p_{1}+1\right)_{n}}{(\Re c+1)_{n}(1)_{n}(2)_{n}}+\sum_{n=0}^{\infty} \frac{\left|(a)_{n}\right|\left|(b)_{n}\right|\left(p_{1}\right)_{n}}{(\Re c)_{n}(1)_{n}(1)_{n}}-1 .
\end{aligned}
$$

An application of Cauchy's inequality gives

$$
\begin{aligned}
S_{2} \leq & \frac{|a b| p_{1}}{\Re c}\left[\left\{\sum_{n=0}^{\infty} \frac{(a+1)_{n}(\bar{a}+1)_{n}\left(p_{1}+1\right)_{n}}{(\Re c+1)_{n}(2)_{n}(1)_{n}}\right\}^{\frac{1}{2}}\left\{\sum_{n=0}^{\infty} \frac{(b+1)_{n}(\bar{b}+1)_{n}\left(p_{1}+1\right)_{n}}{(\Re c+1)_{n}(2)_{n}(1)_{n}}\right\}^{\frac{1}{2}}\right] \\
& +\left\{\sum_{n=0}^{\infty} \frac{(a)_{n}(\bar{a})_{n}\left(p_{1}\right)_{n}}{(\Re c)_{n}(1)_{n}(1)_{n}}\right\}^{\frac{1}{2}}\left\{\sum_{n=0}^{\infty} \frac{(b)_{n}(\bar{b})_{n}\left(p_{1}\right)_{n}}{(\Re c)_{n}(1)_{n}(1)_{n}}\right\}^{\frac{1}{2}}-1 .
\end{aligned}
$$

Since the condition (3.6) is satisfied, the above summations can be written as evaluations of generalized hypergeometric functions and we get

$$
\begin{aligned}
S_{2} \leq & \frac{|a b| p_{1}}{\Re c}\left[\left\{{ }_{3} F_{2}\left(a+1, \bar{a}+1, p_{1}+1 ; \Re c+1,2 ; 1\right)\right\}^{\frac{1}{2}}\left\{{ }_{3} F_{2}\left(b+1, \bar{b}+1, p_{1}+1 ; \Re c+1,2 ; 1\right)\right\}^{\frac{1}{2}}\right] \\
& +\left\{{ }_{3} F_{2}\left(a, \bar{a}, p_{1} ; \Re c, 1 ; 1\right)\right\}^{\frac{1}{2}}\left\{{ }_{3} F_{2}\left(b, \bar{b}, p_{1} ; \Re c, 1 ; 1\right)\right\}^{\frac{1}{2}}-1 .
\end{aligned}
$$

Therefore, in view of (3.9), if the condition (3.7) is satisfied, then $\mathcal{I}_{c}^{a, b}(f) \in$ $\mathcal{R}^{\tau}(A, B)$.

(ii) We follow the lines of proof of (i). In this case we use Lemma 5 (instead of Lemma 3). The proof of Theorem 2 is complete. 
Corollary 3. Let $a \in \mathbb{C} \backslash\{0\}, p_{1}=p_{1}(k)$ be defined by (2.1) and $c \in \mathbb{C}$ satisfy $\Re c>\max \left\{0,2 \Re a+p_{1}\right\}$.

If the hypergeometric inequality

$$
\begin{aligned}
& \frac{|a|^{2} p_{1}}{\Re c}\left\{{ }_{3} F_{2}\left(a+1, \bar{a}+1, p_{1}+1 ; \Re c+1,2 ; 1\right)\right\}+\left\{{ }_{3} F_{2}\left(a, \bar{a}, p_{1} ; \Re c, 1 ; 1\right)\right\} \\
\leq & \frac{(A-B)|\tau|}{1+|B|}+1
\end{aligned}
$$

is satisfied, then $\mathcal{I}_{c}^{a, \bar{a}}$ maps the class $k-\mathcal{S} \mathcal{T}$ into $\mathcal{R}^{\tau}(A, B)$. Further, if

$$
\frac{|a|^{2} p_{1}}{\Re c}\left\{{ }_{3} F_{2}\left(a+1, \bar{a}+1, p_{1}+1 ; \Re c+1,2 ; 1\right)\right\}+\left\{{ }_{3} F_{2}\left(a, \bar{a}, p_{1} ; \Re c, 1 ; 1\right)\right\} \leq 2
$$

is satisfied, then $\mathcal{I}_{c}^{a, \bar{a}}$ maps the class $k-\mathcal{S T}$ into $\mathcal{S} \mathcal{T}$.

Proof. Taking $b=\bar{a}$ in Theorem 2 we get the result.

Corollary 4. Let $a \in \mathbb{C} \backslash\{0\}, p_{1}=p_{1}(k)$ be defined by (2.1) and $c \in \mathbb{C}$ satisfy

$$
\Re c>\max \left\{2+p_{1}, 2 \Re a+p_{1}\right\} \text {. }
$$

If the hypergeometric inequality

$$
\begin{aligned}
& \frac{(\Re c-1)^{\frac{1}{2}}}{\left(\Re c-p_{1}-1\right)^{\frac{1}{2}}}\left[\frac{|a| p_{1}}{\left\{\left(\Re c-p_{1}-2\right) \Re c\right\}^{\frac{1}{2}}}\left\{{ }_{3} F_{2}\left(a+1, \bar{a}+1, p_{1}+1 ; \Re c+1,2 ; 1\right)\right\}^{\frac{1}{2}}\right. \\
& \left.\quad+\left\{{ }_{3} F_{2}\left(a, \bar{a}, p_{1} ; \Re c, 1 ; 1\right)\right\}^{\frac{1}{2}}\right] \\
\leq & \frac{(A-B)|\tau|}{1+|B|}+1
\end{aligned}
$$

is satisfied, then $\mathcal{L}(a, c)$ maps the class $k-\mathcal{S T}$ into $\mathcal{R}^{\tau}(A, B)$. Further, if

$$
\begin{gathered}
\frac{(\Re c-1)^{\frac{1}{2}}}{\left(\Re c-p_{1}-1\right)^{\frac{1}{2}}}\left[\frac{|a| p_{1}}{\left\{\left(\Re c-p_{1}-2\right) \Re c\right\}^{\frac{1}{2}}}\left\{{ }_{3} F_{2}\left(a+1, \bar{a}+1, p_{1}+1 ; \Re c+1,2 ; 1\right)\right\}^{\frac{1}{2}}\right. \\
\left.+\left\{{ }_{3} F_{2}\left(a, \bar{a}, p_{1} ; \Re c, 1 ; 1\right)\right\}^{\frac{1}{2}}\right] \leq 2
\end{gathered}
$$

is satisfied, then $\mathcal{L}(a, c)$ maps the class $k-\mathcal{S T}$ into $\mathcal{S T}$.

Proof. We take $b=1$ in Theorem 2. The Gauss summation formula (1.5) provides the following simplification

$$
\begin{aligned}
{ }_{3} F_{2}\left(2,2, p_{1}+1 ; \Re c+1,2 ; 1\right) & ={ }_{2} F_{1}\left(2, p_{1}+1 ; \Re c+1 ; 1\right) \\
& =\frac{\Gamma(\Re c+1) \Gamma\left(\Re c-p_{1}-2\right)}{\Gamma(\Re c-1) \Gamma\left(\Re c-p_{1}\right)} \\
& =\frac{(\Re c)(\Re c-1)}{\left(\Re c-p_{1}-1\right)\left(\Re c-p_{1}-2\right)}
\end{aligned}
$$


and

$$
\begin{aligned}
{ }_{3} F_{2}\left(1,1, p_{1} ; \Re c, 1 ; 1\right) & ={ }_{2} F_{1}\left(1, p_{1} ; \Re c ; 1\right) \\
& =\frac{\Gamma(\Re c) \Gamma\left(\Re c-p_{1}-1\right)}{\Gamma(\Re c-1) \Gamma\left(\Re c-p_{1}\right)}=\frac{(\Re c-1)}{\left(\Re c-p_{1}-1\right)} .
\end{aligned}
$$

Thus, the conditions (3.7) and (3.8) of Theorem 2 simplify to (3.10) and (3.11) respectively. Therefore, the assertions of Corollary 4 follows from Theorem 2.

Theorem 3. Let $a, b \in \mathbb{C} \backslash\{0\}, p_{1}=p_{1}(k)$ be defined by (2.1) and $c \in \mathbb{C}$ satisfy

$$
\Re c>\max \left\{0,2 \Re a+p_{1}-1,2 \Re b+p_{1}-1\right\} .
$$

(i) If the hypergeometric inequality

$$
\left[{ }_{3} F_{2}\left(a, \bar{a}, p_{1} ; \Re c, 1 ; 1\right)\right]^{\frac{1}{2}}\left[{ }_{3} F_{2}\left(b, \bar{b}, p_{1} ; \Re c, 1 ; 1\right)\right]^{\frac{1}{2}} \leq \frac{(A-B)|\tau|}{1+|B|}+1
$$

is satisfied, then $\mathcal{I}_{c}^{a, b}$ maps the class $k-\mathcal{U C V}$ into the class $\mathcal{R}^{\tau}(A, B)$.

(ii) Furthermore, if

$$
\left[{ }_{3} F_{2}\left(a, \bar{a}, p_{1} ; \Re c, 1 ; 1\right)\right]^{\frac{1}{2}}\left[{ }_{3} F_{2}\left(b, \bar{b}, p_{1} ; \Re c, 1 ; 1\right)\right]^{\frac{1}{2}} \leq 2
$$

is satisfied, then $\mathcal{I}_{c}^{a, b}$ maps the class of $k-\mathcal{U C V}$ into the class $\mathcal{S T}$.

Proof. (i) Let the function $f$ given by (1.1) be a member of $k-\mathcal{U C V}$. We follow the lines of proof of Theorem1. Taking into account the estimates (2.2) for $a_{n}$ and the elementary inequality (3.3), we show that

$$
S_{3}=\sum_{n=2}^{\infty} n \frac{\left|(a)_{n-1}(b)_{n-1}\right|\left(p_{1}\right)_{n-1}}{(\Re c)_{n-1}(1)_{n-1}(1)_{n}} \leq \frac{(A-B)|\tau|}{1+|B|} .
$$

The term $S_{3}$ can be equivalently written as

$$
S_{3}=\sum_{n=1}^{\infty} \frac{\left|(a)_{n}(b)_{n}\right|\left(p_{1}\right)_{n}}{(\Re c)_{n}(1)_{n}(1)_{n}}=\sum_{n=0}^{\infty} \frac{\left|(a)_{n}\right|\left|(b)_{n}\right|\left(p_{1}\right)_{n}}{(\Re c)_{n}(1)_{n}(1)_{n}}-1 .
$$

An application of Cauchy's inequality and the relation

$$
\overline{(d)_{n}}=(\bar{d})_{n}\left(n \in \mathbb{N}_{0}\right)
$$

for any complex number $d$ give

$$
S_{3} \leq\left\{\sum_{n=0}^{\infty} \frac{(a)_{n}(\bar{a})_{n}\left(p_{1}\right)_{n}}{(\Re c)_{n}(1)_{n}(1)_{n}}\right\}^{\frac{1}{2}}\left\{\sum_{n=0}^{\infty} \frac{(b)_{n}(\bar{b})_{n}\left(p_{1}\right)_{n}}{(\Re c)_{n}(1)_{n}(1)_{n}}\right\}^{\frac{1}{2}}-1 .
$$

The conditions $\Re c>2 \Re a+p_{1}-1$ and $\Re c>2 \Re b+p_{1}-1$ given in (3.12) ensure that the sums in the r.h.s of (3.16) are convergent hypergeometric series; so that

$$
S_{3} \leq\left\{{ }_{3} F_{2}\left(a, \bar{a}, p_{1} ; \Re c, 1 ; 1\right)\right\}^{\frac{1}{2}}\left\{{ }_{3} F_{2}\left(b, \bar{b}, p_{1} ; \Re c, 1 ; 1\right)\right\}^{\frac{1}{2}}-1 .
$$


Therefore, in view of (3.15) if the inequality (3.13) is satisfied, then $\mathcal{I}_{c}^{a, b}(f) \in$ $\mathcal{R}^{\tau}(A, B)$ as asserted.

(ii) We follow the lines of proof of (i). In this case we use Lemma 5 (instead of Lemma 3). The proof of Theorem 3 is complete.

Corollary 5. Let the complex numbers $a, b$ and $c$ be as in Theorem 3 and further satisfy the inequality

$$
\left\{{ }_{3} F_{2}\left(a, \bar{a}, p_{1} ; \Re c, 1 ; 1\right)\right\}^{\frac{1}{2}}\left\{{ }_{3} F_{2}\left(b, \bar{b}, p_{1} ; \Re c, 1 ; 1\right)\right\}^{\frac{1}{2}} \leq(1-\beta) \cos \eta+1 .
$$

Then the operator $\mathcal{I}_{c}^{a, b}$ maps $k-\mathcal{U C V}$ into $\mathcal{R}_{\eta}(\beta)$.

Proof. Taking $A=1-2 \beta(0 \leq \beta<1), B=-1$ and $\tau=e^{-i \eta} \cos \eta$ in Theorem $3(\mathrm{i})$ we get the result.

Corollary 6. Let $a \in \mathbb{C} \backslash\{0\}, p_{1}=p_{1}(k)$ be defined by (2.1) and $c \in \mathbb{C}$ satisfy

$$
\Re c>\max \left\{0,2 \Re a+p_{1}-1\right\} .
$$

If the hypergeometric inequality

$$
{ }_{3} F_{2}\left(a, \bar{a}, p_{1} ; \Re c, 1 ; 1\right) \leq \frac{(A-B)|\tau|}{1+|B|}+1
$$

is satisfied, then $\mathcal{I}_{c}^{a, \bar{a}}$ maps the class $k-\mathcal{U C V}$ into $\mathcal{R}^{\tau}(A, B)$. Further, if

$$
{ }_{3} F_{2}\left(a, \bar{a}, p_{1} ; \Re c, 1 ; 1\right) \leq 2
$$

is satisfied, then $\mathcal{I}_{c}^{a, \bar{a}}$ maps the class $k-\mathcal{U C V}$ into $\mathcal{S T}$.

Proof. Taking $b=\bar{a}$ in Theorem 3 we get the result.

Corollary 7. Let $a \in \mathbb{C} \backslash\{0\}, p_{1}=p_{1}(k)$ be defined by (2.1) and $c \in \mathbb{C}$ satisfy

$$
\Re c>\max \left\{2 \Re a+p_{1}-1, p_{1}+1\right\} .
$$

If the hypergeometric inequality

$$
{ }_{3} F_{2}\left(a, \bar{a}, p_{1} ; \Re c, 1 ; 1\right) \leq\left(\frac{(A-B)|\tau|}{1+|B|}+1\right)^{2}\left(\frac{\Re c-p_{1}-1}{\Re c-1}\right)
$$

is satisfied, then $\mathcal{L}(a, c)$ maps $k-\mathcal{U C V}$ into $\mathcal{R}^{\tau}(A, B)$. Further, if

$$
{ }_{3} F_{2}\left(a, \bar{a}, p_{1} ; \Re c, 1 ; 1\right) \leq 4\left(\frac{\Re c-p_{1}-1}{\Re c-1}\right)
$$

is satisfied, then $\mathcal{L}(a, c)$ maps $k-\mathcal{U C V}$ into $\mathcal{S T}$.

Proof. We take $b=1$ in Theorem 3. Note that

$$
\begin{aligned}
{ }_{3} F_{2}\left(1,1, p_{1} ; \Re c, 1 ; 1\right) & ={ }_{2} F_{1}\left(1, p_{1} ; \Re c ; 1\right) \\
& =\frac{\Gamma(\Re c) \Gamma\left(\Re c-p_{1}-1\right)}{\Gamma(\Re c-1) \Gamma\left(\Re c-p_{1}\right)}=\frac{(\Re c-1)}{\left(\Re c-p_{1}-1\right)} .
\end{aligned}
$$

Thus, the conditions (3.13) and (3.14) of Theorem 3 simplify to (3.17) and (3.18) respectively. The assertion of Corollary 7 now follows from Theorem 3. 
Theorem 4. Let $a, b \in \mathbb{C} \backslash\{0\}$ and $c \in \mathbb{C}$ satisfy

$$
\Re c>\max \{0,2 \Re a, 2 \Re b\} .
$$

If the hypergeometric inequality

$$
\frac{\Gamma(\Re c)\{\Gamma(\Re c-2 \Re a) \Gamma(\Re c-2 \Re b)\}^{\frac{1}{2}}}{|\Gamma(\Re c-a)||\Gamma(\Re c-b)|} \leq \frac{1}{1+|B|}+1
$$

is satisfied, then $\mathcal{I}_{c}^{a, b}$ maps the class of $\mathcal{R}^{\tau}(A, B)$ into $\mathcal{R}^{\tau}(A, B)$.

Proof. Let the function $f$ given by (1.1) be a member of $\mathcal{R}^{\tau}(A, B)$. By virtue of Lemma 3 and the coefficient inequality (2.4) it is sufficient to show that

$$
(1+|B|) S_{4} \leq 1,
$$

where

$$
S_{4}=\sum_{n=2}^{\infty} \frac{\left|(a)_{n-1}(b)_{n-1}\right|}{(\Re c)_{n-1}(1)_{n-1}}=\sum_{n=0}^{\infty} \frac{\left|(a)_{n}\right|\left|(b)_{n}\right|}{(\Re c)_{n}(1)_{n}}-1 .
$$

Applications of Cauchy's inequality and the Gauss summation formula (1.5) give

$$
\begin{aligned}
S_{4} & \leq\left\{\sum_{n=0}^{\infty} \frac{(a)_{n}(\bar{a})_{n}}{(\Re c)_{n}(1)_{n}}\right\}^{\frac{1}{2}}\left\{\sum_{n=0}^{\infty} \frac{(b)_{n}(\bar{b})_{n}}{(\Re c)_{n}(1)_{n}}\right\}^{\frac{1}{2}}-1 \\
& =\left\{{ }_{2} F_{1}(a, \bar{a} ; \Re c ; 1)\right\}^{\frac{1}{2}}\left\{{ }_{2} F_{1}(b, \bar{b} ; \Re c ; 1)\right\}^{\frac{1}{2}}-1 \\
& =\frac{\Gamma(\Re c)\{\Gamma(\Re c-2 \Re a) \Gamma(\Re c-2 \Re b)\}^{\frac{1}{2}}}{|\Gamma(\Re c-a)||\Gamma(\Re c-b)|}-1 .
\end{aligned}
$$

Thus, in view of (3.21), if the hypergeometric inequality (3.20) is satisfied, then $\mathcal{I}_{c}^{a, b}(f) \in \mathcal{R}^{\tau}(A, B)$ as asserted. The proof of Theorem 4 is complete.

Corollary 8. Let $a \in \mathbb{C} \backslash\{0\}$ and $c \in \mathbb{C}$ satisfy

$$
\Re c>\max \{0,2 \Re a\} .
$$

If the hypergeometric inequality

$$
\frac{\Gamma(\Re c) \Gamma(\Re c-2 \Re a)}{|\Gamma(\Re c-a)|^{2}} \leq \frac{1}{1+|B|}+1
$$

is satisfied, then $\mathcal{I}_{c}^{a, \bar{a}}$ maps the class of $\mathcal{R}^{\tau}(A, B)$ into itself.

Proof. Taking $b=\bar{a}$ in Theorem 4 we get the result.

Corollary 9. Let $a \in \mathbb{C} \backslash\{0\}$ and $c \in \mathbb{C}$ satisfy

$$
\Re c>\max \{2,2 \Re a\} .
$$

If the hypergeometric inequality

$$
\frac{\Gamma(\Re c)\{\Gamma(\Re c-2 \Re a) \Gamma(\Re c-2)\}^{\frac{1}{2}}}{|\Gamma(\Re c-a)| \Gamma(\Re c-1)} \leq \frac{1}{1+|B|}+1
$$


is satisfied, then $\mathcal{L}(a, c)$ maps $\mathcal{R}^{\tau}(A, B)$ into itself.

Proof. Take $b=1$ in Theorem 4.

Theorem 5. Let $a, b \in \mathbb{C} \backslash\{0\}$ and $c \in \mathbb{C}$ satisfy

$$
\Re c>\max \{0,2 \Re a+1,2 \Re b+1\} .
$$

If the hypergeometric inequality

$$
\begin{aligned}
& \frac{\Gamma(\Re c)\{\Gamma(\Re c-2 \Re a-1) \Gamma(\Re c-2 \Re b-1)\}^{\frac{1}{2}}}{|\Gamma(\Re c-a)||\Gamma(\Re c-b)|}[|a b| \\
& \left.+\{(\Re c-2 \Re a-1)(\Re c-2 \Re b-1)\}^{\frac{1}{2}}\right] \\
\leq & 1+\frac{(A-B)|\tau|}{1+|B|}
\end{aligned}
$$

is satisfied, then $z{ }_{2} F_{1}(a, b ; c ; z) \in \mathcal{R}^{\tau}(A, B)$.

Proof. We know

$$
z_{2} F_{1}(a, b ; c ; z)=\sum_{n=0}^{\infty} \frac{(a)_{n}(b)_{n}}{(c)_{n}(1)_{n}} z^{n+1}=z+\sum_{n=2}^{\infty} \frac{(a)_{n-1}(b)_{n-1}}{(c)_{n-1}(1)_{n-1}} z^{n} .
$$

Therefore, by Lemma 3 , it is sufficient to show that

$$
S_{5}=\sum_{n=2}^{\infty} n \frac{\left|(a)_{n-1} \|(b)_{n-1}\right|}{(\Re c)_{n-1}(1)_{n-1}} \leq \frac{(A-B)|\tau|}{1+|B|} .
$$

As in our demonstration of Theorem 3 we can write $S_{5}$ equivalently as

$$
S_{5}=\frac{|a \| b|}{\Re c} \sum_{n=0}^{\infty} \frac{\left|(a+1)_{n}\right|\left|(b+1)_{n}\right|}{(\Re c+1)_{n}(1)_{n}}+\sum_{n=0}^{\infty} \frac{\left|(a)_{n}\right|\left|(b)_{n}\right|}{(\Re c)_{n}(1)_{n}}-1 .
$$

Applications of Cauchy's inequality followed by the Gauss summation formula (1.5) and the relation $\Gamma(\bar{z})=\overline{\Gamma(z)}$ give

$$
\begin{aligned}
S_{5} \leq & \frac{|a b|}{\Re c}\left[\left\{\sum_{n=0}^{\infty} \frac{(a+1)_{n}(\bar{a}+1)_{n}}{(\Re c+1)_{n}(1)_{n}}\right\}^{\frac{1}{2}}\left\{\sum_{n=0}^{\infty} \frac{(b+1)_{n}(\bar{b}+1)_{n}}{(\Re c+1)_{n}(1)_{n}}\right\}^{\frac{1}{2}}\right] \\
& +\left\{\sum_{n=0}^{\infty} \frac{(a)_{n}(\bar{a})_{n}}{(\Re c)_{n}(1)_{n}}\right\}^{\frac{1}{2}}\left\{\sum_{n=0}^{\infty} \frac{(b)_{n}(\bar{b})_{n}}{(\Re c)_{n}(1)_{n}}\right\}^{\frac{1}{2}}-1 \\
= & \frac{|a b|}{\Re c}\left[\left\{{ }_{2} F_{1}(a+1, \bar{a}+1 ; \Re c+1 ; 1)\right\}^{\frac{1}{2}}\left\{{ }_{2} F_{1}(b+1, \bar{b}+1 ; \Re c+1 ; 1)\right\}^{\frac{1}{2}}\right] \\
& +\left\{{ }_{2} F_{1}(a, \bar{a} ; \Re c ; 1)\right\}^{\frac{1}{2}}\left\{{ }_{2} F_{1}(b, \bar{b} ; \Re c ; 1)\right\}^{\frac{1}{2}}-1 \\
= & \frac{\Gamma(\Re c)\{\Gamma(\Re c-2 \Re a-1) \Gamma(\Re c-2 \Re b-1)\}^{\frac{1}{2}}}{|\Gamma(\Re c-a)||\Gamma(\Re c-b)|} \\
& \times\left[|a b|+\{(\Re c-2 \Re a-1)(\Re c-2 \Re b-1)\}^{\frac{1}{2}}\right]-1 .
\end{aligned}
$$


Thus, in view of (3.24), if the inequality (3.23) is satisfied, then $z_{2} F_{1}(a, b ; c ; z) \in$ $R^{\tau}(A, B)$. The proof of Theorem 5 is complete.

\section{References}

[1] L. de Branges, A proof of the Bieberbach conjecture, Acta Math. 154 (1985), no. 1-2, $137-152$.

[2] T. R. Caplinger and W. M. Causey, A class of univalent functions, Proc. Amer. Math. Soc. 39 (1973), 357-361.

[3] K. K. Dixit and S. K. Pal, On a class of univalent functions related to complex order, Indian J. Pure Appl. Math. 26 (1995), no. 9, 889-896.

[4] P. L. Duren, Univalent Functions, Grundlehren der Mathematischen Wissenschaften [Fundamental Principles of Mathematical Sciences], 259. Springer-Verlag, New York, 1983.

[5] A. Gangadharan, T. N. Shanmugam, and H. M. Srivastava, Generalized hypergeometric functions associated with $k$-uniformly convex functions, Comput. Math. Appl. 44 (2002), no. 12, 1515-1526.

[6] A. W. Goodman, Univalent functions and nonanalytic curves, Proc. Amer. Math. Soc. 8 (1957), 598-601.

[7] - On uniformly convex functions, Ann. Polon. Math. 56 (1991), no. 1, 87-92.

[8] Ju. E. Hohlov, Operators and operations on the class of univalent functions, Izv. Vyssh. Uchebn. Zaved. Mat. 1978 (1978), no. 10, 83-89.

[9] S. Kanas and H. M. Srivastava, Linear operators associated with $k$-uniformly convex functions, Integral Transform. Spec. Funct. 9 (2000), no. 2, 121-132.

[10] S. Kanas and A. Wisniowska, Conic regions and $k$-uniform convexity, J. Comput. Appl. Math. 105 (1999), no. 1-2, 327-336.

[11] _ Conic domains and starlike functions, Rev. Roumaine Math. Pures Appl. 45 (2000), no. 4, 647-657.

[12] W. Ma and D. Minda, Uniformly convex functions, Ann. Polon. Math. 57 (1992), no. $2,165-175$.

[13] K. S. Padmanabhan, On a certain class of functions whose derivatives have a positive real part in the unit disc, Ann. Polon. Math. 23 (1970), 73-81.

[14] S. Ponnusamy and F. Rønning, Starlikeness properties for convolutions involving hypergeometric series, Ann. Univ. Mariae Curie-Sklodowska Sect. A 52 (1998), no. 1, 141-155.

[15] F. Rønning, Uniformly convex functions and a corresponding class of starlike functions, Proc. Amer. Math. Soc. 118 (1993), no. 1, 189-196.

[16] H. M. Srivastava and P. W. Karlson, Multiple Gaussian Hypergeometric Series, Ellis Horwood Series: Mathematics and its Applications. Ellis Horwood Ltd., Chichester; Halsted Press [John Wiley \& Sons, Inc.], New York, 1985.

[17] H. M. Srivastava and A. K. Mishra, Applications of fractional calculus to parabolic starlike and uniformly convex functions, Comput. Math. Appl. 39 (2000), no. 3-4, 5769.

[18] A fractional differintegral operator and its applications to a nested class of multivalent functions with negative coefficients, Adv. Stud. Contemp. Math. (Kyungshang) 7 (2003), no. 2, 203-214.

[19] H. M. Srivastava, A. K. Mishra, and M. K. Das, A nested class of analytic functions defined by fractional calculus, Commun. Appl. Anal. 2 (1998), no. 3, 321-332.

[20] _ A unified operator in fractional calculus and its applications to a nested class of analytic functions with negative coefficients, Complex Variables Theory Appl. 40 (1999), no. 2, 119-132. 
[21] _ A class of parabolic starlike functions defined by means of a certain fractional derivative operator, Fract. Calc. Appl. Anal. 6 (2003), no. 3, 281-298.

Akshaya K. Mishra

Department of Mathematics

BERHAMPUR UNIVERSITY

Berhampur-760007, Orissa, India

E-mail address: akshayam2001@yahoo.co.in

Trailokya Panigrahi

Department of Mathematics

Templecity Institute of Technology and Engineering

F/12, IID CENTRE

Knowledge Campus

Barunei, Khurda, 752057, Orissa, India

E-mail address: t_panispce@rediffmail.com 